SMAD, Rev. Eletrônica Saúde Mental Álcool Drog.

2019 jul.-set.;15(3):1-9

DOI: 10.11606/issn.1806-6976.smad.2019.000403

www.revistas.usp.br/smad/

Artigo Original

\title{
Consumo de álcool e drogas e participação em violência por adolescentes de uma região trinacional
}

\section{Elis Maria Teixeira Palma Priotto ${ }^{1}$ \\ (D) https://orcid.org/0000-0003-1949-2183 Marta Angelica lossi Silva² \\ (D) https://orcid.org/0000-0002-9967-8158}

Objetivo: identificar a associação entre o consumo de drogas e álcool e a participação em violência de adolescentes da tríplice fronteira, de três municípios/países: Brasil, Paraguai e Argentina. Método: estudo descritivo transversal com 2788 adolescentes estudantes, com amostragem aleatória. Resultados: consumo elevado de tabaco e álcool nos três municípios/países. As meninas consomem mais álcool, adolescentes argentinos consomem mais drogas. Os paraguaios como vítimas tem maiores chances para o consumo de álcool e drogas, os brasileiros como vítimas e vítimas/agressores tendem para o consumo de bebidas alcoólicas. Conclusão: a exposição à violência entre os adolescentes é alta quando é associada ao uso de álcool. Há a necessidade de intervenções como prevenção e educação em saúde nas escolas de uma região trinacional.

Descritores: Violência; Adolescente; Áreas de Fronteira; Consumo de Bebidas Alcoólicas; Drogas Ilícitas.

\section{Como citar este artigo}

Priotto EMTP, Silva MAI. Consumption of alcohol and drugs and youth violence in a tri-national region. SMAD, Rev Eletrônica Saúde Mental Álcool Drog. 2019;15(3):1-9. doi: https://dx.doi.org/10.11606/issn.1806-6976.smad.2019.000403 


\title{
Consumption of alcohol and drugs and youth violence in a tri-national region
}

\begin{abstract}
Objective: to identify the association between consumption of drugs and alcohol and the violence amongst teenagers of the frontier of Brazil, Paraguay and Argentina. Method: cross-sectional descriptive study, with 2788 students teenagers, with random sampling. Results: the heightened consumption of tobacco and alcohol in the three mentioned cities/countries. Girls consume more alcohol. Argentinian teenagers consume more drugs. Paraguayans as victims have increased chances of consumption of alcohol and drugs, and, brazilians as victims and victims/aggressors by consumption of alcohol. Conclusion: that the exposition of violence between teenagers is high, and is associated with the use of alcohol. Interventions such as prevention and health education in schools of a tri-national frontier region are needed.
\end{abstract}

Descriptors: Violence; Teenager; Border Areas; Alcohol Drinking; Street Drugs.

\section{Consumo de alcohol y drogas y participación en violencia por adolescentes de una región trinacional}

Objetivo: identificar la asociación entre el consumo de drogas y alcohol y la participación en violencia de adolescentes de tres municipios/ países: Brasil, Paraguay y Argentina. Método: estudio descriptivo transversal con 2788 adolescentes, con muestreo aleatorio. Resultados: consumo elevado de tabaco y alcohol en los tres municipios/países. Las niñas consumen más alcohol, los adolescentes argentinos consumen más drogas. Los paraguayos como víctimas tienen más posibilidades para el consumo de alcohol y drogas, los brasileños como víctimas y víctimas/agresores propenden para el consumo de bebidas alcohólicas. Conclusión: altos índices de violencia asociados al uso de alcohol. Hay la necesidad de intervenciones como prevención y educación en salud en las escuelas de una región trinacional.

Descritores: Violência; Adolescente; Áreas Fronterizas; Consumo de Bebidas Alcoholicas; Drogas Ilícitas. 


\section{Introdução}

A violência e o consumo de drogas são temas recorrentes no universo da literatura, e a associação do consumo de drogas lícitas e ilícitas com alterações de comportamento e práticas violentas é considerada, na sociedade atual, um problema de saúde pública. Estudos constataram uma ligação entre o consumo de álcool e comportamentos violentos ${ }^{(1-3)}$, porém este estudo faz a associação entre o consumo de álcool, outras drogas e a participação em violência entre adolescentes de três municípios/países.

Há mais de duas décadas, a violência é reconhecida pela a Organização Mundial de Saúde e pelo Ministério de Saúde do Brasil como grave problema de saúde pública, bem como constitui uma questão da pauta dos movimentos globais por saúde e da agenda de saúde

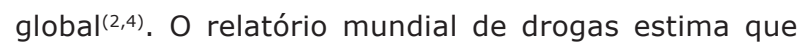
um em cada 20 adultos ou um quarto de bilhão de pessoas, com idade entre 15 e 64 anos, usaram pelo menos uma droga ilícita durante sua vida. Além disso, há a estimativa de que 76,3 milhões de pessoas possuam diagnóstico de consumo abusivo de álcool(5).

De modo geral, o problema de saúde tem como causas relacionadas ao consumo de álcool e outras drogas, o aumento de comportamentos violentos, o aumento do narcotráfico, o uso da violência por grupos organizados para a dominação e controle territorial sejam estes resultantes de tráfico, de milícias, nas áreas de biopirataria, nos municípios de fronteira na rota do tráfico, ou do contrabando de armas e produtos ${ }^{(4,6)}$.

Neste sentido, ao considerar o cenário de região de fronteira trinacional, ou seja,Foz do Iguaçu no Brasil, Ciudad del Este no Paraguai e Puerto Iguazú na Argentina, existe a referência aos municípios conhecidos por serem atípicos ao refletirem uma série de problemas sociais. Entre os problemas, há principalmente a relação a ações ilegais que na última década, transformou-se na principal rota internacional de financiamento e tráfico de drogas, de armas, de seres humanos, de órgãos, do crime organizado, do terrorismo, violências e de contrabando da América do Sul. Ressalta-se que o Brasil efetua a distribuição para o mundo, enquanto o Paraguai é reconhecido como o maior produtor de maconha e também por ser rota de passagem de cocaína da Colômbia, da Bolívia e de outros países ${ }^{(5)}$.

Com isso, justifica-se o interesse em desenvolver um estudo com adolescentes por serem indivíduos que passam por várias alterações físicas e psíquicas, que se expõem a situações de violência e comportamentos de risco como o uso/consumo de álcool e outras drogas. Ademais, a associação desses fatores torna-se motivo de preocupação por se constituir numa tendência crescente de vulnerabilidade para os adolescentes ${ }^{(3,7-8)}$.
Dada a escassez de estudos científicos sobre violência e uso de drogas por adolescentes na região de fronteira do Brasil, Paraguai e Argentina, este trabalho se propôs a identificar a associação entre o consumo de drogas e álcool e a participação em violência de adolescentes de três municípios: fronteira do Brasil, Paraguai e Argentina.

\section{Método}

Foi realizado um estudo de corte transversal analítico, entre 2012 a 2013, em três municípios/países Puerto Iguazú (Argentina), Foz do Iguaçu (Brasil) e Ciudad del Este (Paraguai), com adolescentes estudantes do Ensino Fundamental ( 70 ao 90 ano) e Ensino Médio (10 a 30 ano) de escolas públicas e residentes nos municípios de Foz do Iguaçu (Brasil/BRA), Ciudad del Este (Paraguai/PAR) e Puerto Iguazú (Argentina/ARG).

Utilizou-se um questionário, semiestruturado, autoaplicável e anônimo, elaborado pelos pesquisadores, o qual foi traduzido, analisado e avaliado em cada município/país por dois profissionais da área da educação. Realizou-se um pré-teste com 10 alunos de cada município/país, totalizando 30 participantes voluntários.

Analisaram-se as questões relativas às características sociodemográficas de sexo, idade e país, bem como, os tipos de drogas (lícitas e ilícitas) e bebidas alcoólicas que o adolescente consumiu nos últimos 30 dias. Nas questões específicas de violências criou-se uma categorização entendida como participação em violência dos adolescentes que se encaixavam vítima $(V)$, agressor $(A)$, vítima/agressor $(V / A)$, nem vítima nem agressor (NVNA), seriam os observadores em relação ao consumo de drogas e bebidas alcoólicas nos últimos 30 dias.

O tamanho amostral obteve-se considerando o total de alunos matriculados em escolas e colégios públicos nos três municípios/países. Tendo como critérios de inclusão adolescentes com idade entre 12 e 18 anos de ambos os sexos e residentes no município/país em estudo. A dificuldade de compreensão e interpretação das perguntas do questionário foi utilizada como critério de exclusão dos adolescentes.

Usou-se o método de amostra aleatória estratificada, por meio de um sorteio com base nas listas fornecidas pelas escolas/colégios, totalizando 2.788 adolescentes, sendo 1.014 adolescentes de Puerto Iguazú (ARG), 1.071 adolescentes de Foz do Iguaçu (BRA) e 703 adolescentes de Ciudad del Este (PAR).

$\mathrm{Na}$ coleta de dados em cada instituição de ensino, os pesquisadores procuraram os estudantes em sala de aula, apresentaram a proposta do estudo e os convidaram a participar. No dia subsequente, para a aplicação do questionário foi restrito aos alunos convidados 
(sorteados) e que trouxeram o termo de consentimento assinado pelo responsável e do assentimento assinado pelo adolescente. Na sequência, fez-se a leitura do questionário entregue para resposta dos estudantes, na presença do pesquisador no ambiente escolar.

A análise dos dados foi precedida pela elaboração de um banco de dados no programa Excel e depois importados para a análise estatística no programa Statistical Analysis Software (SAS), versão 9.0, considerando-se um nível de significância em todas as análises de 95\%. Para a descrição geral dos dados relativos às características sociodemográficas, obteve-se a prevalência por meio de análise descritiva de frequência absolutas e relativas. Utilizou-se o modelo de regressão logística que foi ajustado para medir e identificar os fatores associados ao sexo, país, participantes da violência e consumo de drogas e álcool, segundo a medida de quantificação da associação do Odds Ratio/ Razão de Chances (OR). Sendo definido como referência para as respostas afirmativas (Sim), o sexo feminino (F) e o país Brasil (BRA). O estudo foi aprovado pelo Comitê de Ética em Pesquisa (CEP) da Universidade Estadual do Oeste do Paraná- UNIOESTE (Parecer 357/2011-CEP).

\section{Resultados}

Dos 2.788 adolescentes estudantes entrevistados evidenciou-se a idade média de 14,6 anos, sendo $50,71 \%$ do sexo feminino e $45,22 \%$, do sexo masculino. O sexo feminino mostrou-se estatisticamente significativo $(O R=M / F=0,47)$, para o consumo de bebidas alcoólicas, em relação ao sexo masculino. Os adolescentes brasileiros apresentaram uma associação significativa $(O R=A R G / B R A=0,71)$ de maiores chances para o consumo de drogas em relação aos adolescentes Argentinos. Enquanto os adolescentes paraguaios apresentaram uma associação estatisticamente significativa $(O R=P A R / B R A=2,66)$ de maiores chances para o consumo de drogas e de bebidas alcoólicas $(\mathrm{OR}=2,00)$, em relação aos adolescentes do Brasil (Tabela 1).

O tabaco foi a droga mais consumida pelos adolescentes estudantes nos três municípios/países. Os adolescentes da Argentina apresentaram resultados significativos estatisticamente de maiores chances $(O R=1,58)$ para o tabaco $(O R=1,42)$, maconha $(\mathrm{OR}=4,27)$ e crack, em relação aos adolescentes do Brasil. Por outro lado, os adolescentes do Brasil em relação aos do Paraguai obtiveram maiores chances para o consumo do tabaco $(O R=0,43)$, maconha $(O R=0,18)$, cocaína $(O R=0,26)$ e outras drogas $(O R=0,12)$. O Brasil em relação a Argentina obteve maiores chances de consumo para outras drogas $(O R=A R G / B R A=0,10)$. Por sexo, o masculino apresentou associação significativa para o consumo do tabaco $(O R=1,24)$, maconha $(O R=2,29)$, cocaína $(O R=2,14)$, anabolizantes $(O R=3,75)$ e êxtases $(O R=3,85)$. O sexo feminino mostrou associação significativa para o consumo de remédio para emagrecer (R.E), (OR=M/F=0,40) (Tabela 2).

Quanto ao consumo de bebidas alcoólicas evidenciou-se por país, que os adolescentes do Brasil apresentaram associação estatisticamente significativa para o consumo de cerveja, aguardente (pinga, cachaça), vodca, uísque e espumante em relação aos adolescentes da Argentina e do Paraguai. Os adolescentes da Argentina mostraram uma associação $(O R=A R G / B R A=1,22)$ de maiores chances em relação ao Brasil, para consumir vinho $(\mathrm{OR}=\mathrm{ARG} / \mathrm{BRA}=2,81) \mathrm{e}$ para 'outras' bebidas como Gancia, (bebida de origem americana feita com frutas cítricas e açúcar) o Frizze (vinho mesclado espumante com frutas tropicais) e o Frezze (vinho encorpado, com alto teor alcoólico). Por sexo, o percentual demonstra que ambos consomem bebidas alcoólicas. Tendo o sexo feminino maiores chances $(\mathrm{OR}=\mathrm{F} / \mathrm{M}=0,85)$ para o consumo de vinho em relação ao sexo masculino (Tabela 3 ).

Tabela 1 - Consumo de drogas e bebidas alcoólicas pelos estudantes adolescentes nos últimos 30 dias, em relação ao sexo e país: Argentina, Brasil e Paraguai, 2013

\begin{tabular}{|c|c|c|c|c|c|c|c|c|}
\hline \multirow{3}{*}{ Consumo } & \multicolumn{2}{|c|}{ Sexo } & \multirow{3}{*}{$\frac{\text { OR/Sexo }}{\text { MvsF }}$} & \multicolumn{3}{|c|}{ País } & \multicolumn{2}{|c|}{ OR/País } \\
\hline & $\mathbf{F}$ & M & & ARG & BRA & PAR & ARG/BRA & PAR/BRA \\
\hline & $n(\%)$ & $n(\%)$ & & $n(\%)$ & $n(\%)$ & $n(\%)$ & OR(IC95\%) & OR(IC95\% \\
\hline \multicolumn{9}{|l|}{ Drogas } \\
\hline Sim & $237(16,76)$ & $257(20,38)$ & \multirow{3}{*}{$\begin{array}{c}1,19 \\
(0,78-1,81)\end{array}$} & $244(24,06)$ & $210(19,61)$ & $57(8,11)$ & \multirow{3}{*}{$\begin{array}{c}0,71 \\
(0,59-0,87)\end{array}$} & \multirow{3}{*}{$\begin{array}{c}2,66 \\
(2,01-3,53)\end{array}$} \\
\hline Não & $1122(79,35)$ & $954(75,65)$ & & $711(70,12)$ & $819(76,47)$ & $631(89,76)$ & & \\
\hline NR & $55(3,89)$ & $50(3,97)$ & & $59(5,82)$ & $42(3,92)$ & $15(2,13)$ & & \\
\hline \multicolumn{9}{|c|}{ Bebidas Alcoólicas } \\
\hline Sim & $949(67,11)$ & $803(63,67)$ & \multirow{3}{*}{$\begin{array}{c}0,47 \\
(0,24-0,89)\end{array}$} & $713(70,32)$ & $736(68,72)$ & $373(53,06)$ & \multirow{3}{*}{$\begin{array}{c}0,84 \\
(0,69-1,01)\end{array}$} & \multirow{3}{*}{$\begin{array}{c}2,00 \\
(1,64-2,43)\end{array}$} \\
\hline Não & $450(31,82)$ & $431(34,18)$ & & $274(27,02)$ & $230(29,88)$ & $325(46,23)$ & & \\
\hline NR & $15(1,06)$ & $27(2,14)$ & & $27(2,66)$ & $15(1,40)$ & $5(0,71)$ & & \\
\hline
\end{tabular}


Tabela 2 - Distribuição por tipos de drogas usadas/consumidas pelos adolescentes em relação ao sexo e país: Argentina, Brasil e Paraguai, 2013

\begin{tabular}{|c|c|c|c|c|c|c|c|c|c|}
\hline \multirow{3}{*}{$\begin{array}{l}\text { Tipos de } \\
\text { Drogas }\end{array}$} & \multirow{3}{*}{ Consumo } & \multicolumn{2}{|c|}{ Sexo } & \multirow[t]{2}{*}{ OR/ Sexo } & \multicolumn{3}{|c|}{ País } & \multicolumn{2}{|c|}{ OR/ País } \\
\hline & & M & $\mathrm{F}$ & & ARG & BRA & PAR & ARG/BRA & PAR/BRA \\
\hline & & $n(\%)$ & $n(\%)$ & MvsF & $n(\%)$ & $n(\%)$ & $n(\%)$ & OR(IC95\%) & OR(IC95\%) \\
\hline & Sim & $202(16,02)$ & $188(13,30)$ & 1,24 & $207(20,41)$ & $149(13,91)$ & $46(6,54)$ & 1,58 & 0,43 \\
\hline \multirow[t]{3}{*}{ Tabaco } & Não & $1059(83,98)$ & $1226(86,70)$ & $(1,00-1,54)$ & $807(79,59)$ & $922(86,09)$ & $657(93,46)$ & $(1,26-1,99)$ & $(0,30-0,61)$ \\
\hline & NR & 113 & & & & & & & \\
\hline & Sim & $98(7,77)$ & $50(3,54)$ & 2,29 & $83(8,19)$ & $63(5,88)$ & $8(1,14)$ & 1,42 & 0,18 \\
\hline \multirow[t]{3}{*}{ Maconha } & Não & $1163(92,23)$ & $1364(96,46)$ & $(1,62-3,26)$ & $931(91,81)$ & $1008(94,12)$ & $695(98,86)$ & $(1,01-2,00)$ & $(0,08-0,38)$ \\
\hline & NR & 113 & & & & & & & \\
\hline & Sim & $45(3,57)$ & $24(1,70)$ & 2,14 & $23(2,27)$ & $39(3,64)$ & $7(1)$ & 0,61 & 0,26 \\
\hline \multirow[t]{3}{*}{ Cocaína } & Não & $1216(96,43)$ & $1390(98,30)$ & $(1,29-3,53)$ & $991(97,73)$ & $1032(96,36)$ & 696(99) & $(0,36-1,03)$ & $(0,11-0,59)$ \\
\hline & NR & 113 & & & & & & & \\
\hline & Sim & $14(1,11)$ & $8(0,57)$ & 1,97 & $16(1,58)$ & $4(0,37)$ & $2(0,28)$ & 4,27 & 0,76 \\
\hline \multirow[t]{3}{*}{ Crack } & Não & $1247(98,89)$ & $1406(99,43)$ & $(0,82-4,71)$ & $998(98,42)$ & $1067(99,63)$ & $701(99,72)$ & $(1,42-12,83)$ & $(0,13-4,16)$ \\
\hline & NR & 113 & & & & & & & \\
\hline & Sim & $10(0,79)$ & $3(0,21)$ & 3,75 & 10(0.99) & $3(0,28)$ & 0 & 3,54 & * \\
\hline \multirow[t]{3}{*}{ Anabolizantes } & Não & $1251(99,21)$ & $1411(99,79)$ & $(1,03-13,68)$ & $1004(99,01)$ & $1068(99,72)$ & $7039(100)$ & $(0,97-12,92)$ & \\
\hline & NR & 113 & & & & & & & \\
\hline & Sim & $12(0,95)$ & $33(2,33)$ & 0,40 & $18(1,78)$ & $17(1,59)$ & $12(1,71)$ & 1,12 & 1,07 \\
\hline \multirow[t]{3}{*}{${ }^{* \star *}$ R.E } & Não & $1249(99,05)$ & $1381(97,67)$ & $(0,20-0,78)$ & $996(98,22)$ & $1054(98,41)$ & $691(98,29)$ & $(0,57-2,18)$ & $(0,51-2,26)$ \\
\hline & NR & 113 & & & & & & & \\
\hline & Sim & $17(1,35)$ & $5(0,35)$ & 3,85 & $7(0,69)$ & $10(0,93)$ & $5(0,71)$ & 0,73 & 0,76 \\
\hline \multirow[t]{3}{*}{ Êxtase } & Não & $1244(98,65)$ & $1409(99,65)$ & $(1,41-10,46)$ & $1007(99,31)$ & $1061(99,07)$ & $698(99,29)$ & $(0,28-1,94)$ & $(0,25-2,23)$ \\
\hline & NR & 113 & & & & & & & \\
\hline & Sim & $36(2,85)$ & $31(2,19)$ & 1,31 & $6(0,59)$ & $58(5,41)$ & $5(0,71)$ & 0,10 & 0,12 \\
\hline \multirow[t]{3}{*}{ Outros } & Não & $1225(97,15)$ & $1383(97,81)$ & $(0,80-2,13)$ & $1008(99,41)$ & $1013(94,58)$ & $698(99,29)$ & $(0,04-0,24)$ & $(0,05-0,31)$ \\
\hline & $\mathrm{NR}$ & 113 & & & & & & & \\
\hline & Sim & $286(22,68)$ & $364(25,74)$ & 0,84 & $209(20,61)$ & $298(27,82)$ & $171(24,32)$ & 0,67 & 0,83 \\
\hline \multirow[t]{2}{*}{ Nunca usei } & Não & $975(77,32)$ & $1050(74,26)$ & $(0,70-1,01)$ & $805(79,39)$ & $773(72,18)$ & $532(75,68)$ & $(0,55-0,82)$ & $(0,67-1,03)$ \\
\hline & NR & 113 & & & & & & & \\
\hline
\end{tabular}

MvsF = masculino versus feminino. O feminino é a referência; $*$ Dados insuficientes

IC = intervalo de confiança; $\mathrm{LI}=$ limite inferior; $\mathrm{LS}=$ limite superior; $\mathrm{NR}=\mathrm{Não}$ Respondeu $* * * \mathrm{RE}=$ Remédio para Emagrecer; OR = Odds Ratio; $A R G=$ Argentina; BRA = Brasil; PAR = Paraguai

Tabela 3 - Distribuição por tipos de bebidas alcoólicas consumidas nos últimos 30 dias pelos adolescentes em relação ao sexo e país: Argentina, Brasil e Paraguai, 2013

\begin{tabular}{|c|c|c|c|c|c|c|c|c|c|}
\hline \multirow{3}{*}{$\begin{array}{l}\text { Bebidas } \\
\text { Alcoólicas }\end{array}$} & \multirow{3}{*}{ Consumo } & \multicolumn{2}{|c|}{ Sexo } & \multirow[t]{2}{*}{ OR/Sexo } & \multicolumn{3}{|c|}{ País } & \multicolumn{2}{|c|}{ OR/ País } \\
\hline & & $\mathbf{M}$ & $\mathbf{F}$ & & ARG & BRA & PAR & ARG/BRA & PAR/BRA \\
\hline & & $n(\%)$ & $n(\%)$ & MvsF & $n(\%)$ & $n(\%)$ & $n(\%)$ & OR(IC95\%) & OR(IC95\%) \\
\hline & Sim & $681(54)$ & $681(48,16)$ & 0,96 & $505(49,80)$ & $586(54,72)$ & $230(32,72)$ & 0,81 & 0,40 \\
\hline \multirow[t]{3}{*}{ Cerveja } & Não & $732(51,77)$ & $732(51,77)$ & $(0,82-1,11)$ & $509(50,20)$ & $484(45,19)$ & $473(67,28)$ & $(0,68-0,97)$ & $(0,32-0,48)$ \\
\hline & NR & 113 & & & & & & & \\
\hline & Sim & $138(9,76)$ & $141(11,18)$ & 1,16 & $96(9,47)$ & $176(16,43)$ & $14(1,99)$ & 0,53 & 0,10 \\
\hline \multirow[t]{3}{*}{ ACP } & Não & $1276(90,24)$ & $1120(88,20)$ & $(0,90-1,49)$ & $918(90,53)$ & $895(83,57)$ & $689(98,01)$ & $(0,40-0,69)$ & $(0,05-0,18)$ \\
\hline & NR & 113 & & & & & & & \\
\hline & Sim & $273(21,65)$ & $310(21,92)$ & 0,98 & $251(24,75)$ & $312(29,13)$ & $39(5,55)$ & 0,80 & 0,14 \\
\hline \multirow[t]{3}{*}{ Vodca } & Não & $988(78,35)$ & $1104(78,08)$ & $(0,81-1,18)$ & $763(75,25)$ & $759(70,87)$ & $664(94,45)$ & $(0,65-0,97)$ & $(0,10-0,20)$ \\
\hline & NR & 113 & & & & & & & \\
\hline & Sim & $278(22,05)$ & $309(21,85)$ & 1,01 & $212(20,91)$ & $327(30,53)$ & $69(9,82)$ & 0,60 & 0,24 \\
\hline \multirow[t]{3}{*}{ Uísque } & Não & $983(77,95)$ & $1105(78,08)$ & $(0,84-1,21)$ & $802(79,09)$ & $744(69,47)$ & $634(90,18)$ & $(0,49-0,73)$ & $(0,18-0,32)$ \\
\hline & NR & 113 & & & & & & & \\
\hline & Sim & $455(36,08)$ & $563(39,82)$ & 0,85 & $463(45,66)$ & $436(40,71)$ & $158(22,48)$ & 1,22 & 0,42 \\
\hline \multirow[t]{3}{*}{ Vinho } & Não & $806(63,92)$ & $851(60,18)$ & $(0,73-0,99)$ & $551(53,34)$ & $635(59,29)$ & $545(77,52)$ & $(1,02-1,45)$ & $(0,34-0,52)$ \\
\hline & NR & 113 & & & & & & & \\
\hline & Sim & $224(17,76)$ & $280(19,80)$ & 0,87 & $169(16,67)$ & $243(22,69)$ & $109(15,50)$ & 0,68 & 0,62 \\
\hline \multirow[t]{3}{*}{ Espumante } & Não & $1037(82,24)$ & $1134(80,20)$ & $(0,72-1,06)$ & $845(83,33)$ & $828(77,31)$ & $594(84,50)$ & $(0,54-0,84)$ & $(0,48-0,80)$ \\
\hline & NR & 113 & & & & & & & \\
\hline & Sim & $106(8,41)$ & $99(7)$ & 1,21 & $134(13,21)$ & $55(5,14)$ & $24(3,41)$ & 2,81 & 0,65 \\
\hline \multirow[t]{2}{*}{ Outros } & Não & $1155(91,59)$ & 1315(93) & $(0,91-1,62)$ & $880(86,79)$ & $1016(94,86)$ & $679(96,59)$ & $(2,02-3,89)$ & $(0,40-1,06)$ \\
\hline & NR & 113 & & & & & & & \\
\hline
\end{tabular}

*Dados insuficientes; **IC = intervalo de confiança; OR = Odds Ratio; LI = limite inferior; LS = limite Superior; MvsF = Masculino versus feminino; NR = Não Respondeu; $\mathrm{ACP}=$ água ardente/cachaça/pinga; $\mathrm{ARG}=$ Argentina; $\mathrm{BRA}=$ Brasil; PAR = Paraguai 
Na Tabela 4, aparece a categorização dos participantes da violência como vítimas $(V)$, agressores $(A)$, vítimas e agressores ( $V / A)$, e nem vítimas e nem agressores (NVNA). Nesta, os resultados evidenciaram que os participantes da violência totalizaram $37,59 \%$ vítimas, $4,80 \%$ agressores, $31,56 \%$ vítima/agressor e 22,67\%, nem vítima nem agressor (os observadores) (dados não mostrados). Os Adolescentes do Paraguai como vítimas apareceram com associação significativa de maiores chances (OR=PAR/ $B R A=1,68)$ para consumirem drogas licitas e ilícitas em relação aos adolescentes da Argentina e do Brasil.
Na Tabela 5, o conjunto de dados sugere que a relação dos adolescentes do Brasil como participantes da violência para consumirem bebidas alcoólicas se revelaram com maiores chances como vítimas (OR=PAR/ $B R A=0,57)$ e vítimas/agressores ( $O R=P A R / B R A=0,45)$, em relação aos adolescentes do Paraguai. Os adolescentes da Argentina evidenciaram percentagem mais elevada para consumo de bebidas alcoólicas nos últimos 30 dias, enquanto participantes da violência como agressores e vítimas/agressores.

Tabela 4 - Caracterização dos adolescentes participantes da violência por consumo de drogas com relação ao país: Argentina, Brasil e Paraguai, 2013

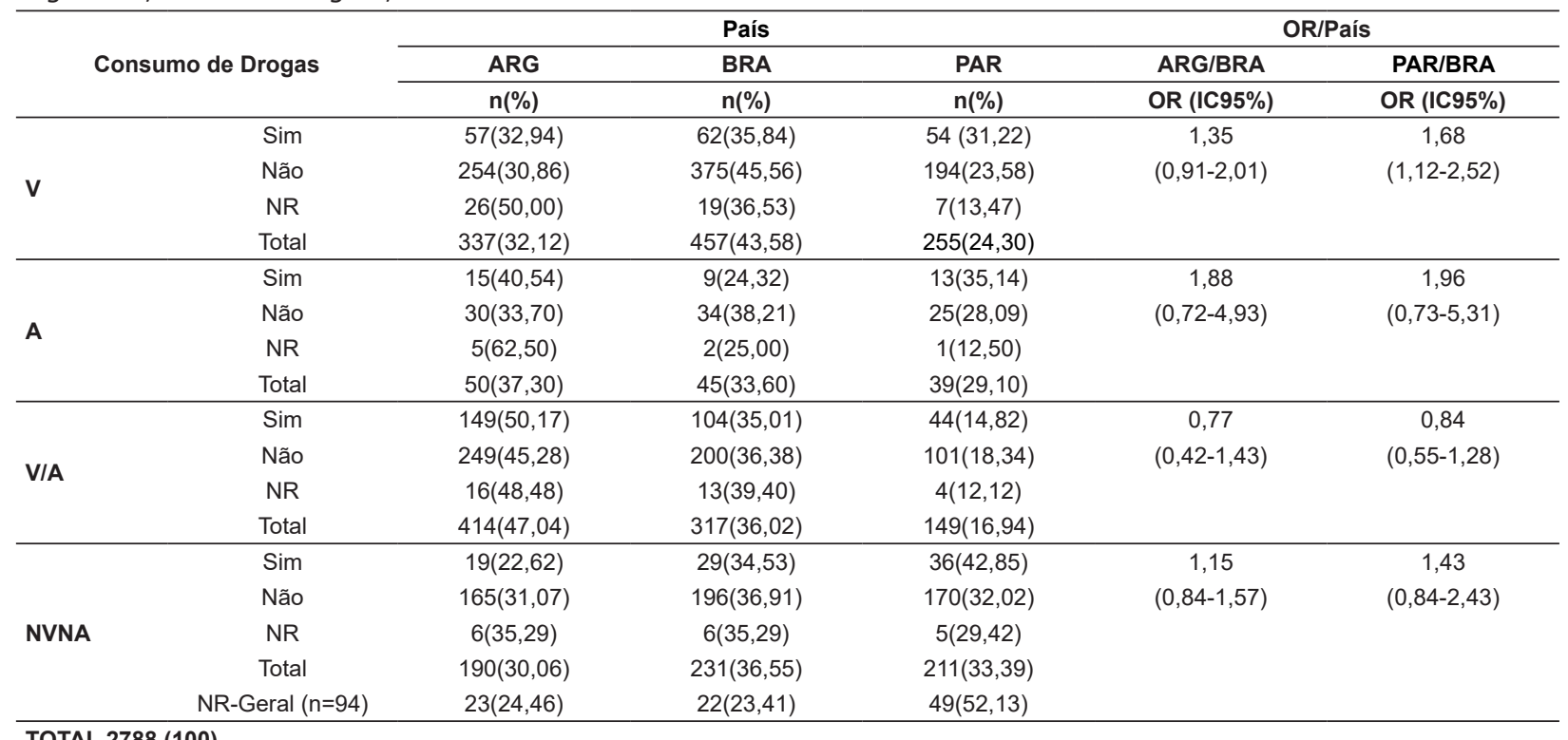

\section{TOTAL 2788 (100)}

IC = Intervalo de Confiança; LI = Limite Inferior; LS = Limite Superior; NR = Não Respondeu; OR = Odds Ratio; ARG = Argentina; BRA = Brasil; PAR = Paraguai; $\mathrm{V}=$ Vítimas; $\mathrm{A}=$ Agressores; $\mathrm{V} / \mathrm{A}=$ Vítimas e Agressores; NVNA = Nem Vítimas e Nem Agressores

Tabela 5 - Caracterização dos adolescentes participantes da violência por consumo de bebidas alcoólicas com relação ao país: Argentina, Brasil e Paraguai, 2013

\begin{tabular}{|c|c|c|c|c|c|c|}
\hline & & \multicolumn{3}{|c|}{ País } & \multicolumn{2}{|c|}{ OR/País } \\
\hline \multicolumn{2}{|c|}{ Consumo de Bebidas Alcoólicas } & ARG & BRA & PAR & ARG/BRA & PAR/BRA \\
\hline & & $\mathrm{n}(\%)$ & $\mathrm{n}(\%)$ & $\mathrm{n}(\%)$ & OR(IC95\%) & OR(IC95\%) \\
\hline \multirow{4}{*}{$\mathbf{v}$} & Sim & $217(32,72)$ & $306(46,13)$ & $141(21,15)$ & 0,93 & 0,57 \\
\hline & Não & $108(29,58)$ & $143(39,16)$ & $114(31,26)$ & $(0,69-1,27)$ & $(0,42-0,79)$ \\
\hline & NR & $12(63,16)$ & $7(36,84)$ & $0(0)$ & & \\
\hline & Total & $337(32,12)$ & $457(43,57)$ & $255(24,31)$ & & \\
\hline \multirow{4}{*}{ A } & Sim & $41(36,94)$ & $39(35,14)$ & $31(27,92)$ & 0,75 & 0,49 \\
\hline & Não & $7(35)$ & $5(25)$ & $8(40)$ & $(0,22-2,56)$ & $(0,14-1,67)$ \\
\hline & NR & $2(66,67)$ & $1(33,33)$ & $0(0)$ & & \\
\hline & Total & $50(37,30)$ & $45(33,60)$ & $39(29,10)$ & & \\
\hline \multirow{4}{*}{ V/A } & Sim & $339(48,22)$ & $255(36,27)$ & $109(15,51)$ & 1,02 & 0,45 \\
\hline & Não & $67(40,61)$ & $60(36,36)$ & $38(23,03)$ & $(0,69-1,51)$ & $(0,31-0,67)$ \\
\hline & NR & $8(66,66)$ & $2(16,67)$ & $2(16,67)$ & & \\
\hline & Total & $414(47,04)$ & $317(36,02)$ & $149(16,94)$ & & \\
\hline \multirow{5}{*}{ NVNA } & Sim & $105(34,20)$ & $126(41,04)$ & $76(24,76)$ & 1,19 & 0,67 \\
\hline & Não & $82(25,71)$ & $101(32,06)$ & $133(42,23)$ & $(0,81-1,74)$ & $(0,42-1,07)$ \\
\hline & NR & $3(33,33)$ & $4(44,44)$ & $2(22,23)$ & & \\
\hline & Total & $190(30,06)$ & $231(36,55)$ & $211(33,39)$ & & \\
\hline & NR-Geral $(n=94)$ & $23(24,47)$ & $22(23,40)$ & $49(52,13)$ & & \\
\hline
\end{tabular}

IC = intervalo de confiança; LI = Limite Inferior; LS = Limite Superior; NR = Não Respondeu; OR = Odds Ratio; ARG = Argentina; BRA = Brasil; PAR = Paraguai 


\section{Discussão}

Este é o primeiro estudo de base populacional, com inquérito escolar de adolescentes alunos na faixa etárias de 12 a 18 anos, residentes em municípios de fronteira da Argentina, Brasil e Paraguai, cujos resultados sugerem uma associação entre o consumo de drogas e bebidas alcoólicas e o aumento das probabilidades de produção da violência dos adolescentes dos três municípios/países. O sexo masculino destaca-se com maiores chances para o consumo de drogas ilícitas, enquanto o sexo feminino mostrou percentagens de maior consumo para bebidas alcoólicas.

Entre as drogas lícitas mais consumidas pelos adolescentes por municípios/países, surge o tabaco, tendo a Argentina a maior taxa percentual. E, por sexo, a predominância é do sexo masculino, discordando de outro estudo que aponta a prevalência $(22,3 \%)$ de consumo do tabaco é pelo sexo feminino, com idade média de 16 anos $^{(5)}$. Sabe-se que o uso do tabaco é um problema de saúde pública no mundo, anualmente registra-se $25 \%$ da população, ou seja, cinco milhões de mortes no mundo em consequência de doenças causados pelo tabagismo(9).

A segunda droga mais consumida pelos adolescentes com predominância pelo sexo masculino, nos três municípios/países e no mundo é a maconha

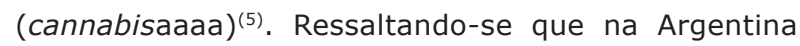
é permitido o consumo como pessoal e o Paraguai atualmente é o maior produtor de maconha da América Latina(9).

O consumo da cocaína aparece como a terceira mais consumida pelos adolescentes do Brasil e da Argentina nos últimos trinta dias. As demais substâncias psicoativas como anabolizantes, êxtases e o crack, são mais procuradas, pois no mercado mundial mais que dobrou o consumo, entre 2009 e 2013, evidenciando maior preocupação na América Latina e no Caribe ${ }^{(5)}$.

Cerca de seis milhões de pessoas (4\% da população adulta) já experimentaram alguma vez na vida a cocaína ou a droga fumada: o crack e o óxi. Entre os adolescentes, 442 mil (3\% dos que têm entre 14 anos e 18 anos) também já tiveram experiência com algum tipo dessas substâncias ${ }^{(1,11)}$. Na América do Sul, em especial no Brasil, o consumo e o tráfico de cocaína cresceram ${ }^{(5)}$. Na última década, as apreensões da polícia federal de Foz do Iguaçu (Brasil) têm mostrado o aumento do tráfico de drogas como a cocaína e o crack, e mais recentemente de drogas sintéticas na tríplice fronteira. E a maconha passou a ter menor importância no ranking das drogas que entram no território brasileiro via Foz do Iguaçu, fronteira Paraguai para Brasil(14).

O sexo feminino, no presente estudo mostrou-se na preferência pelo consumo de remédio para emagrecer $(\mathrm{RE})$, sendo as anfetaminas e tranquilizantes as mais usadas. Comenta-se que a nível mundial o número de consumidores dessas drogas ficou entre 125 e 203 milhões de pessoas, representando uma taxa de $2,8 \%$ para $4,5 \%$ de prevalência. Indica-se que a preferência para o uso das anfetaminas, ocorre devido à sua popularidade, além da sua relativa facilidade de produção e disponibilidade nos municípios/países, o que facilita a sua aquisição e experimentação( ${ }^{(9)}$ e maior propensão de iniciar precocemente, evoluindo para dependência(1,10-11).

Nesse sentido, o estudo exposto, entre outros confirmam que o consumo de drogas licitas e ilícitas, tem apresentado aumento na população brasileira e da Argentina em geral. Com especificidade para o sexo masculino, além da sua associação com diversos agravos à saúde(4-5).

O presente estudo também evidenciou dados significativos para o consumo de álcool pelo sexo feminino, divergindo de outras pesquisas realizadas por estudos nacionais e internacionais ao referir a predominância do sexo masculino para o uso de álcool e drogas lícitas e ilícitas ${ }^{(2,10,12)}$. Entretanto, estudos corroboram ao confirmar que o consumo de bebidas alcoólicas é de uma prevalência significativa para as meninas $^{(4)}$. Contudo, os resultados aqui mostrados em percentuais coincidem com os demais estudos por citar a preferência dos adolescentes de ambos os sexos para o consumo de tabaco e álcool(4-5,9,14), destaca-se ainda que o álcool, é a substância psicotrópica considerada droga legal, mais utilizada por adolescentes no Brasil e no mundo. Seu consumo nessa pesquisa é preocupante, tanto pela maior tendência à impulsividade e atividades de risco, nessa fase da vida, quanto pelo dano ao desenvolvimento cerebral na adolescência, determinando repercussões durante a vida adulta(5).

Os resultados aqui apresentados sugerem ainda, que os adolescentes do Brasil e participantes da violência como vítimas e vítimas/agressores e consomem mais bebidas alcoólicas que os adolescentes vítimas e vítimas/ agressores do Paraguai. Enquanto os adolescentes do Paraguai e participantes da violência como vítimas e têm mais chances de consumirem drogas lícitas e ilícitas em relação aos adolescentes da Argentina e do Brasil.

Esta constatação é preocupante e é confirmada em um estudo realizado também em escolas, que os adolescentes apresentavam maior agressividade e desatenção aos estudos devido ao uso de álcool e outras drogas, sugerindo-se que o consumo de álcool e outras drogas torna-os suscetíveis às influencias de amigos, bem como o seu envolvimento em outros comportamentos de risco. O uso/consumo retroalimenta a violência praticada por ambos os $\operatorname{sexos}^{(4)}$.

Desta forma, o indicativo é a busca de intervenções preventivas destinadas aos adolescentes quanto ao consumo de álcool e drogas e as vulnerabilidades e/ou 
comportamentos de risco possíveis, bem como ofertar orientações urgentes e necessárias sobre como lidar, evitar e desenvolver estratégias adequadas.

Assim, diante dos resultados da análise de regressão logística, que revelam a associação do consumo de álcool e demais drogas são fatores de vulnerabilidade que interferem estatisticamente na participação de ações de violências. Isso tudo vidência um impacto negativo na saúde individual e coletiva, tanto do sexo masculino como no feminino.

Dados da Organização Mundial de Saúde (OMS) (5) revelam a magnitude do problema social e apontam que $25 \%$ de todas as mortes de jovens entre 15 e 19 anos são atribuídas ao consumo de álcool. Essas estatísticas refletem o padrão de consumo de álcool por adolescentes ${ }^{(8)}$, que reconhecem os riscos entre o consumo de álcool, as alterações de comportamento e as violências, com maior prevalência e risco para o sexo feminino ${ }^{(2,14)}$.

Ademais, os dados confirmados sugerem por municípios/países que os adolescentes brasileiros apresentaram maiores chances de consumir drogas em relação aos adolescentes Argentinos. Porém, foram os adolescentes paraguaios que se destacaram com maiores chances para consumirem drogas ilícitas e lícitas e bebidas alcoólicas em relação aos adolescentes do Brasil.

No intuito de compreender melhor os resultados achados acerca da participação na violência envolvendo adolescentes vítimas, agressores, vítimas/agressores ou nem vítimas nem agressores, tanto nos cenários do Brasil do Paraguai e da Argentina e sugere-se que mesmo não obtendo resultados estatisticamente comprobatórios e todos têm chances para consumirem drogas e álcool e serem participantes em violências. Essa associação é confirmada em outros estudos que evidenciam o aumento da violência frente ao uso de drogas e bebidas alcoólicas ${ }^{(1,5,13)}$.

Atenta-se para o fato de que o consumo de drogas e bebidas alcoólicas pelos adolescentes dos três municípios/países, é um fator preocupante e com destaque os índices elevados de consumo de álcool nos últimos 30 dias, que interferem e aumentam a vulnerabilidade dos adolescentes como participantes da violência. Evidência essa, que contradiz o fato de que por ser uma região de fronteira a prevalência do uso de drogas ilícitas seria significativa.

\section{Conclusão}

Observa-se que os dados aqui mostrados reforçam que a região de fronteira entre o Brasil e o Paraguai se constitui em uma importante região de passagem de tráfico de drogas, atividade esta que potencializa o uso/ consumo de drogas e a criminalidade, uma complexa rede ilegal neste território. No entanto, ao contrário do esperado, evidenciou-se que apesar dos adolescentes residirem em municípios de fronteira, com elevado índice de apreensão de drogas, tráfico e criminalidade, estes apresentaram uma taxa menor no consumo de drogas ilícitas e uma associação estatisticamente significativa para o consumo de álcool e o tabaco, drogas lícitas, de baixo custo e acesso facilitado, o que favorece a aceitação social, sendo esses, adolescentes, participantes da violência na tríplice fronteira.

Pode-se dizer então, que diante dos dados aqui analisados se faz necessário intervir com ações de educação em saúde e a inclusão desses temas no currículo das escolas e colégios, sobre a prevenção. Se o consumo de drogas e a produção da violência receberem a atenção necessária dos professores e profissionais da saúde. Como fatores proeminentes, na concepção trinacional agravada em contextos específicos, é preciso olhar as diversidades culturais, as desigualdades, as iniquidades, as exclusões, as negligências, e à inoperância dos governos no fortalecimento de vínculos entre ciência e política de modo que os resultados de pesquisas promissoras sejam identificadas, sintetizadas e comunicadas de forma eficaz para os políticos e ao público, rompendo com a imagem de que a violência entre adolescentes é algo comum e esperado.

Este estudo tem algumas limitações sobre as análises, devido a amostra selecionada de acordo com a participação em violência e o consumo de álcool e outras drogas. Assim, os resultados podem referir-se apenas para os padrões de comportamento dessa amostra. No entanto, é importante considerar que o estudo foi realizado em três municípios de fronteira e assim devendo-se ponderar as diferenças geográficas e sociais neste domínio.

\section{Referências}

1. Elicker E, Palazzo LS, Aerts DRGC, Alves GG, et. al. Uso de álcool, tabaco e outras drogas por adolescentes escolares de Porto Velho-RO, Brasil. Epidemiol Serv Saúde . [Internet]. 2015 Set [Acesso 16 jun 2017]; 24(3): 399-410. Disponível em: http://scielo.iec. pa.gov.br/scielo.php?script=sci_arttext\&pid=S167949742015000300006\&lng=pt.

2. Singh V, Epstein NQ, Cunningham RM, Stoddard SA, et. Physical dating violence among adolescents and young adults with alcohol misuse. Drug Alcohol Depend. 2015;153:364-8. Available from: https://www.ncbi.nlm. nih.gov/pmc/articles/PMC4509806.

3. Reis DC, Almeida TAC, Miranda MM, Alves $\mathrm{RH}$, et al. Health vulnerabilities in adolescence: socioeconomic conditions, social networks, drugs and violence. Rev. Latino-Am. Enfermagem. [Internet]. 2013. Apr [cited 2017 June 16]; 21(2):586-94. Available 
from: http://www.scielo.br/scielo.php?script=sci_ arttext\&pid $=$ S0104-11692013000200586\&Ing=en.

4. Malta DC, Mascarenhas MDM, Porto DL, Barreto SM, et al. Exposição ao álcool entre escolares e fatores associados. Rev Saúde Pública. [Internet]. 2014. Feb [Acesso 16 jun 2017]; 48 (1): 52-62. Disponível em: http://www.scielo.br/scielo.php?script=sci arttext\&pid=S0034-89102014000100052\&lng.

5. UNODC- United Nations Office on Drugs and Crime. World Drug Report. United Nations. New York; 2016.

6. Organización Panamericana de la Salud. Organización Mundial de Informe de situación regional sobre el alcohol y la salud en las Américas. Washington, DC: OPS; 2015. 7. Correas A, Rodriguez HS, Cuesta P, López-CE, et al. Exploratory Analysis of Power Spectrum and Functional Connectivity During Resting State in Young Binge Drinkers: A MEG Study. Int J Neural Systems. 2015;25(3):1550008.

8. Gemelli V, Souza EBC. Fronteiras Brasil/Paraguai e as Redes de Drogas Ilícitas. Rev ANPEGE. 2012;8(10):101-13.

9. Wagner GA, Oliveira LG, Barroso LP, Nishimura R, et al. Drug use in college students: a 13-year trend. Rev Saúde Pública. [Internet]. 2012. June [cited em 2017 June 16]; 46(3): 497-504. Available from: http://www. scielo.br/scielo.php?script=sci_arttext\&pid=S003489102012000300012\&lng=en.Epub Apr 24, 2012.

10. Observatorio Europeo de las Drogas y las Toxicomanias. Informe sobre los mercados de drogas en la EU. Lisboa, Portugal; 2016.

11. Ministério da Saúde (BR). Secretaria Nacional de Políticas sobre drogas. Estimativa do número de usuários de crack e/ou similares nas capitais do país. Brasília: Ministérios da Saúde; 2013.

12. Carneiro CP. Fronteira Irmãs: transfronteirizações na Bacia do Prata. Porto Alegre: Ed. Ideograf; 2016. 273p. 13. Souza AM, Barufaldi LA, Abreu GA, Giannini D, et al. ERICA: ingestão de macro e micronutrientes em adolescentes brasileiros. Rev Saúde Pública. [Internet]. 2016[Acesso em 2017 June16];50(Suppl1):5s. Disponível em: http://www.scielo.br/scielo.php?script=sci_arttext\& pid $=$ S003489102016000200309\&

en. Epub Feb 23, 2016.

14. Puente LAR, Castilho BAA, Castilho MMA, Castilho MTA, et al. Consumo álcool e tabaco entre os adolescentes. SMAD, Rev Eletrônica Saúde Mental Álcool Drog. 2016;12(4):200-6. doi: https://doi.org/10.11606/ issn. 1806-6976.v12i4p200-206
Recebido: 13.07.2017

Aceito: 31.07 .2018
Copyright $\odot 2019$ SMAD, Rev. Eletrônica Saúde Mental Álcool Drog. Este é um artigo de acesso aberto distribuído sob os termos da Licença Creative Commons CC BY-NC.

Esta licença permite que outros remixem, adaptem e criem a partir do seu trabalho para fins não comerciais, e embora os novos trabalhos tenham de lhe atribuir o devido crédito e não possam ser usados para fins comerciais, os usuários não têm de licenciar esses trabalhos derivados sob os mesmos termos. 